\title{
Pathogenic mutations identified by a multimodality approach in 117 Japanese Fanconi anemia patients
}

\begin{abstract}
Minako Mori, ${ }^{1,2}$ Asuka Hira, ${ }^{1}$ Kenichi Yoshida, ${ }^{3}$ Hideki Muramatsu, ${ }^{4}$ Yusuke Okuno, ${ }^{4}$ Yuichi Shiraishi, ${ }^{5}$ Michiko Anmae, ${ }^{6}$ Jun Yasuda, ${ }^{7}$ Shu Tadaka, ${ }^{7}$ Kengo Kinoshita,,${ }^{7,8}$ Tomoo Osumi, ${ }^{10}$ Yasushi Noguchi, ${ }^{11}$ Souichi Adachi, ${ }^{12}$ Ryoji Kobayashi, ${ }^{13}$ Hiroshi Kawabata, ${ }^{14}$ Kohsuke Imai, ${ }^{15}$ Tomohiro Morio, ${ }^{16}$ Kazuo Tamura, ${ }^{6}$ Akifumi Takaori-Kondo, ${ }^{2}$ Masayuki Yamamoto, ${ }^{7,17}$ Satoru Miyano, ${ }^{5}$ Seiji Kojima, ${ }^{4}$ Etsuro Ito, ${ }^{18}$ Seishi Ogawa, ${ }^{3,19}$ Keitaro Matsuo, ${ }^{20}$ Hiromasa Yabe, ${ }^{21}$ Miharu Yabe $^{21}$ and Minoru Takata ${ }^{1}$

${ }^{1}$ Laboratory of DNA Damage Signaling, Department of Late Effects Studies, Radiation Biology Center, Graduate School of Biostudies, Kyoto University, Kyoto, Japan; '2Department of Hematology and Oncology, Graduate School of Medicine, Kyoto University, Kyoto, Japan; ${ }^{3}$ Department of Pathology and Tumor Biology, Graduate School of Medicine, Kyoto University, Kyoto, Japan; ${ }^{4}$ Department of Pediatrics, Nagoya University Graduate School of Medicine, Nagoya, Japan; ${ }^{5}$ Laboratory of DNA Information Analysis, Human Genome Center, The Institute of Medical Science, University of Tokyo, Tokyo Japan; ${ }^{\circ}$ Medical Genetics Laboratory, Graduate School of Science and Engineering, Kindai University, Osaka, Japan; ${ }^{7}$ Tohoku Medical Megabank Organization, Tohoku University, Sendai, Japan; ${ }^{8}$ Department of Applied Information Sciences, Graduate School of Information Sciences, Tohoku University, Sendai, Japan; Institute of Development, Aging, and Cancer, Tohoku University, Sendai, Japan; ${ }^{10}$ Children's Cancer Center, National Center for Child Health and Development, Tokyo, Japan; ${ }^{11}$ Department of Pediatrics, Japanese Red Cross Narita Hospital, Chiba, Japan; ${ }^{12}$ Department of Pediatrics, Kyoto University Graduate School of Medicine, Kyoto, Japan; ${ }^{13}$ Department of Pediatrics and Adolescence, Sapporo Hokuyu Hospital, Sapporo, Japan; ${ }^{14}$ Department of Hematology and Immunology, Kanazawa Medical University, Uchinada-machi, Japan; ${ }^{15}$ Department of Community Pediatrics, Perinatal and Maternal Medicine, Tokyo Medical and Dental University, Tokyo, Japan; ${ }^{16}$ Department of Pediatrics and Developmental Biology, Tokyo Medical and Dental University, Tokyo, Japan; ${ }^{17}$ Department of Medical Biochemistry, Graduate School of Medicine, Tohoku University, Sendai, Japan; ${ }^{18}$ Department of Pediatrics, Hirosaki University Graduate School of Medicine, Hirosaki, Japan; ${ }^{19}$ Department of Medicine, Center for Hematology and Regenerative Medicine, Karolinska Institute, Stockholm, Sweden; ${ }^{20}$ Division of Molecular and Clinical Epidemiology, Aichi Cancer Center Research Institute, Nagoya, Japan and ${ }^{21}$ Department of Innovative Medical Science, Tokai University School of Medicine, Isehara, Kanagawa, Japan
\end{abstract}

An incorrected version of table 2 appared On October 2019 Issue, page 1967. The corrected version of table 2 is published on the next page.

Table 2. Clinical phenotype of 10 Japanese Fanconi anemia patients with VACTERL-H association.

\begin{tabular}{|c|c|c|c|c|c|c|c|c|}
\hline Individual & $\begin{array}{l}\text { Affected } \\
\text { gene }\end{array}$ & $\begin{array}{l}\text { Mutation } \\
\text { patterns }\end{array}$ & $\begin{array}{l}\text { VACTERL-H } \\
\text { features }\end{array}$ & FA-features & $\begin{array}{l}\text { Family } \\
\text { history } \\
\text { of FA* }\end{array}$ & $\begin{array}{l}\text { Birth } \\
\text { weight SD } \\
\text { score }\end{array}$ & $\begin{array}{c}\text { DEB } \\
\text { induced } \\
\text { chromosome } \\
\text { breakage } \\
\text { (breaks / cell) }\end{array}$ & $\begin{array}{l}\text { ALDH2 *** } \\
\text { genotype } \\
\text { ) }\end{array}$ \\
\hline Case 18-1 & FANCA & $\begin{array}{l}\text { c.4042_4043insC: } \\
\text { p.I1348TfsX77 }\end{array}$ & $\begin{array}{l}\text { C: PDA } \\
\text { R: Left renal agenesis } \\
\text { L: Bilateral absent thumbs/ } \\
\text { Bilateral radial hypoplasia }\end{array}$ & $\begin{array}{l}\text { Short } \\
\text { stature }\end{array}$ & + & -1.9 & 0.44 & $\mathrm{AA}$ \\
\hline Case 30 & FANCA & $\begin{array}{l}\text { c.2546delC: } \\
\text { p.S849FfsX40 }\end{array}$ & $\begin{array}{c}\text { V: scoliosis } \\
\text { C: ASD/Persistent left superior vena } \\
\text { E: Esophageal atresia }\end{array}$ & $\begin{array}{l}\text { Skin pigmentation } \\
\text { Deafness } \\
\text { Right inguinal hernia } \\
\text { Bicornuate uterus } \\
\text { Short stature (-1.8SD) }\end{array}$ & - & -2.1 & 2.06 & GG \\
\hline Case 37 & FANCA & $\begin{array}{l}\text { c.2546delC: } \\
\text { p.S849FfsX40 }\end{array}$ & $\begin{array}{c}\text { E: Esophageal atresia } \\
\text { R: Right pelvic kidney } \\
\text { L: Bilateral thumb hypoplasia }\end{array}$ & $\begin{array}{c}\text { Jejunal atresia } \\
\text { Strabismus } \\
\text { Short stature (-4SD) }\end{array}$ & - & -2.3 & 0.12 & $\mathrm{GG}$ \\
\hline
\end{tabular}


continued from previous page

\begin{tabular}{|c|c|c|c|c|c|c|c|c|}
\hline Case 60 & FANCB & $\begin{array}{c}\text { complete loss } \\
\text { of FANCB gene } \\
\text { rrX g.14730104-14904216 del) }\end{array}$ & $\begin{array}{l}\text { V: Spina bifida occulta/ } \\
\text { Abnormal ribs } \\
\text { A: Anal atresia } \\
\text { C: PDA } \\
\text { R: Right renal agenesis } \\
\text { L: Right absent thumb/ } \\
\text { Partial loss of left thumb }\end{array}$ & $\begin{array}{c}\text { Skin pigmentation } \\
\text { Microphthalmus/ } \\
\text { Stenocephaly/Ptosis } \\
\text { Duodenal stenosis } \\
\text { Annular pancreas/ } \\
\text { Hypospadias/ } \\
\text { Undescended testis } \\
\text { Short stature (-6SD) }\end{array}$ & - & -4.8 & 3.8 & $\mathrm{GG}$ \\
\hline Case 61 & $\begin{array}{r}F A N C B \\
\quad(\mathrm{c}\end{array}$ & $\begin{array}{c}\text { complete loss } \\
\text { of } F A N C B \text { gene } \\
\text { rrX g.14810970-14932973 del) }\end{array}$ & $\begin{array}{l}\text { V: Abnormal ribs/Scoliosis } \\
\text { A: Anal atresia } \\
\text { C: VSD/PS } \\
\text { E: Duodenal atresia** } \\
\text { R: Left renal agenesis } \\
\text { L: Bilateral absent thumbs } \\
\text { H: Hydrocephalus }\end{array}$ & $\begin{array}{c}\text { Skin pigmentation } \\
\text { Microphthalmus/ } \\
\text { Deafness/ Ear canal stenosis } \\
\text { Undescended testis } \\
\text { (Short stature (-1SD)) }\end{array}$ & - & -2.8 & 4.2 & $\mathrm{GA}$ \\
\hline Case 64 & FANCC & c. $1154+5 \mathrm{G}>\mathrm{A}:$ p.S386X & $\begin{array}{c}\text { A: Anal atresia } \\
\text { C: VSD, PDA } \\
\text { E: Esophageal atresia }\end{array}$ & $\begin{array}{c}\text { Skin pigmentation } \\
\text { Deafness/Left aural stenosis/ } \\
\text { Right aural atresia } \\
\text { Cleft palate } \\
\text { Short stature (-2SD) }\end{array}$ & - & -2.53 & 7.8 & $\mathrm{GG}$ \\
\hline Case 69 & FANCG & $\begin{array}{c}\text { c. } 307+1 \mathrm{G}>\mathrm{C} \\
\text { c.1066C }>\text { T: p.Q356X }\end{array}$ & $\begin{array}{l}\text { C: Coarctation complex } \\
\text { R: Right renal agenesis/ } \\
\text { Left renal cyst } \\
\text { L: Bilateral absent thumbs/ } \\
\text { Right radial hypoplasia }\end{array}$ & $\begin{array}{l}\text { Skin pigmentation } \\
\text { Short stature (-8SD) }\end{array}$ & - & -1.7 & 8.54 & $\mathrm{GA}$ \\
\hline Case 73-1 & FANCG & $\begin{array}{l}\text { c. } 307+1 \mathrm{G}>\mathrm{C} \\
\text { c. } 307+1 \mathrm{G}>\mathrm{C}\end{array}$ & $\begin{array}{c}\text { C: PDA } \\
\text { R: Left renal agenesis } \\
\text { L: Right absent thumb/ } \\
\text { Bilateral radial hypoplasia }\end{array}$ & $\begin{array}{c}\text { Skin pigmentation } \\
\text { Bilateral aural atresia } \\
\text { Short stature (-2.7SD) }\end{array}$ & + & -0.9 & 3.49 & GA \\
\hline Case 96 & FANCI & c.288G>A:p.C56FfsX8 & $\begin{array}{c}\text { A: Anal atresia } \\
\text { C: VSD/PDA } \\
\text { R: Right renal agenesis/ } \\
\text { Left renal hypoplasia } \\
\text { L: Bilateral absent thumb/ } \\
\text { Bilateral absent radius } \\
\text { H: Hydrocephalus }\end{array}$ & $\begin{array}{l}\text { Skin pigmentation } \\
\text { Microphthalmus } \\
\text { Hypogenitalia } \\
\text { Short stature (-8SD) }\end{array}$ & - & -3.9 & 0.52 & $\mathrm{GA}$ \\
\hline Case 99-1 & FANCP & $\begin{array}{c}\text { c.343delA: } \\
\text { p.S115AfsX11 }\end{array}$ & $\begin{array}{c}\text { C: ASD/NSD/PS } \\
\text { R: horseshoe kidney } \\
\text { L: Bilateral floating thumbs/ } \\
\text { bilateral radial hypoplasia }\end{array}$ & $\begin{array}{l}\text { Intestinal malrotation } \\
\text { Duodenal stenosis } \\
\text { Short stature (-5.8SD) }\end{array}$ & + & -2.3 & 0.91 & $\mathrm{AA}$ \\
\hline
\end{tabular}

${ }^{*}$ Case 18-1,73-1, and 99-1 had a sibling with Fanconi anemia (FA). ** Duodenal atresia is considered to be a part of the VACTERL association by some reports. ${ }^{27} * * * A L D H 2$ wild type and the inactivating mutation (p.Glu504Lys) allele is referred to as G and A, respectively. ALDH2: aldehyde dehydrogenase-2; ASD: atrial septal defect; BM: bone marrow; DEB: diepoxybutane; PDA: patent ductus arteriosus; PS: pulmonary stenosis; SD: Standard Deviation;VACTERL-H: vertebral anomalies, anal atresia, cardiac anomalies, trachealesophageal fistula, esophageal atresia, renal structural abnormalities, limb anomalies, and hypocephalus; VSD: ventricular septal defect. 